\title{
Influence of a Static Magnetic Field on Beam Emittance in Laser Wakefield Acceleration
}

\author{
Mathieu Drouin, Alain Bourdier, Quentin Harry, Sébastien Rassou \\ CEA, DAM, DIF, Arpajon, France \\ Email: mathieu.drouin@cea.fr
}

Received September 19, 2012; revised November 2, 2012; accepted November 10, 2012

\begin{abstract}
The enhancement of trapping and the optimization of beam quality are two key issues of Laser Wakefield Acceleration (LWFA). The effect of a homogenous constant magnetic field $B_{0}$, parallel to the direction of propagation of the pump pulse, is studied in the blowout regime via 2Dx3Dv Particle-In-Cell simulations. Electrons are injected into the wake using a counter-propagating low amplitude laser. Transverse currents are generated at the rim of the bubble, which results in the amplification of the $B_{0}$ field at the rear of the bubble. Therefore the dynamics of the beam is modified, the main effect is the reduction of the transverse emittance when $B_{0}$ is raised. Depending on beam loading effects the low energy tail, observed in the non-magnetized case, can be suppressed when $B_{0}$ is applied, which provides a mono-energetic beam.
\end{abstract}

Keywords: Laser Wakefield Acceleration; Magnetic Field; Beam Emittance; Beam Loading

\section{Introduction}

In Laser Wake-Field Acceleration (LWFA) [1-4], a laser creates a plasma wave wakefield with a phase velocity close to the speed of light $(c)$. The acceleration gradients in these wakefields can easily exceed $100 \mathrm{GeV} / \mathrm{m}$, hence a cm-long plasma based accelerator can produce$\mathrm{GeV}$-energy electron beams. An electron injected in such a wave gains energy from the longitudinal component of the electric field, as long as the pump pulse is not depleted and the dephasing length is not reached. These wakefields have ideal properties for accelerating electrons. The transverse focusing field increases linearly with the radial distance and the accelerating longitudinal field is independent of the radial coordinate [5,6]. LWFA can be split into different options. The first corresponds to a plasma density $n_{e} \approx 10^{19} \mathrm{~cm}^{-3}$, a pulse length $(c \tau)$ matching half of a plasma period and a spot size $\left(w_{0}\right)$ roughly equals to the bubble radius, $w_{0} \approx c \tau \approx \sqrt{a_{0}}$, where $a_{0}$ is the normalized vector potential of the laser. This is the idea of the bubble regime [7,8]. For these conditions, a hundred-joule class laser would have an intensity of the order $\sim 10^{21} \mathrm{~W} \cdot \mathrm{cm}^{-2}$. In this regime, the electrons are continuously injected, this results in tremendous beam loading and the loaded wake is noisy. In this paper electron injection is achieved using the colliding pulse scheme $[9,10]$, hence the bubble regime is not appropriate. We rather select moderate laser intensity
$I \leq 10^{19} \mathrm{~W} \cdot \mathrm{cm}^{-2}$ and plasma density $n_{e} \leq 10^{18} \mathrm{~cm}^{-3}$ according to the guidelines proposed by $\mathrm{Lu}$ et al. [11] to achieve a more controlled and stable blowout of the electrons, with no self-injection. In order to limit the computational requirements of our PIC (Particle-In-Cell) simulations the propagation of the pump pulse will not exceed 1 $\mathrm{cm}$, moreover we restrict our study to $2 \mathrm{Dx} 3 \mathrm{Dv}$ (two- dimensional space and three-dimensional velocity). We focus on wakefield acceleration in the presence of an external, initially homogenous, magnetic field and we study its influence through PIC simulations. In the first part of this paper, we will give the simulation setup and we will specify how the plasma is magnetized. Then, in the second part, the main findings induced by the magnetic field will be detailed. Attention will be paid to self-consistent amplification of the magnetic field at the rear of the bubble. The emittance of the accelerated beam will be compared with or without $B_{0}$ field. In the third part, the influence of the magnetic field on the energy distribution of the beam will be briefly commented. Lastly we will draw some conclusions.

\section{Simulation Setup}

The effect of a strong external magnetic field has seldom been reported in the context of wakefield acceleration. Let us first summarize some recent papers. It was first proposed in the context of LWFA by Hur et al. [12], with a single pump laser $a_{0}=3.5$ and an electronic density $n_{e}$ $=3 \times 10^{-3} n_{c}$, where $n_{c}$ is the critical density. This setup is 
prone to self-injection into the wakefield, therefore the quality of the accelerated beam is quite degraded [12]. Recently Vieira et al. [13] have considered magnetic fields oriented perpendicularly to a laser or particle beam driver. They showed that this magnetic field configuration can relax the self-trapping thresholds, leading to off axis self-injection with narrow transverse trapping crosssections.

Our simulation setup is distinct from these two latter. Here we aim at studying the influence of an external magnetic field, oriented along the laser propagation direction, in the blowout regime [11] with colliding pulse injection of the electrons [9]. Hence we chose $a_{0}=4$ and $n_{e}=4.4 \times 10^{17} \mathrm{~cm}^{-3}=2.5 \times 10^{-4} n_{c}$, thus abiding by $a_{0} \geq 4$ and $2 \leq a_{0} \leq 2 \omega_{0} / \omega_{p}$ criteria proposed by Martins et al. [14]. The required magnetic field necessary to curve electron trajectories is about a hundred teslas [12], such values are particularly strong but still available from the current pulsed magnet technology [15], the most advanced magnets can reach $90 \mathrm{~T}$ for tens of ms durations and centimeter size lengths [16]. The simulation setup consists in two 30 fs linearly polarized counter propagating waves with $\lambda=0.8 \mu \mathrm{m}$ wavelength. They propagate along a constant homogeneous guide field $B_{0}$ in a cmlong plasma, the normalized value of $B_{0}$ is given by $\tilde{B}_{0}=e B_{0} /\left(m_{e} \omega_{0}\right)$. Their electric fields are in the sameplane (P linear polarizations). The pump pulse, whichcreates the accelerating wakefield, is focused to an 18 $\mu \mathrm{m}$ full width at half maximum. The low intensity pulse is focused to a $31 \mu \mathrm{m}$ focal spot at a peak normalized vector potential $a_{1}=0.1$. Electrons will be considered trapped in the bubble when their Lorentz factor exceeds $\gamma_{\text {las }}$, defined by

$$
\gamma_{\text {las }}=\left[1-\left(\frac{v_{g}}{c}\right)^{2}\right]^{-1 / 2}
$$

where $v_{g}$ is the group velocity of the laser. In the present case we get $\gamma_{\text {las }} \approx 63$. The group velocity is related to the plasma density via

$$
\left(\frac{v_{g}}{c}\right)=\left(1-\frac{\omega_{p}^{2}}{\omega_{0}^{2}}\right)^{1 / 2},
$$

where $\omega_{p}$ and $\omega_{0}$ respectively denote the plasma and laser frequencies. Let us now specify how the plasma is magnetized. As our simulations use the moving frame technique, consisting in a window sliding at the group velocity of the laser, we have to initialize fresh plasma, at each time step, on the receiving border of the simulation box. The value of the magnetic field is chosen static and uniform in the slice where the plasma is initialized. Note that the value of the magnetic field is the solution of Maxwell's equations anywhere else in the simulation box, which means that the physics of LWFA will modify the magnetic field.

\section{A New Mechanism to Enhance the Beam Emittance}

We have checked that no electron is trapped into the wake when there is no colliding pulse and $B_{0}=0$, $125 \mathrm{~T}$ or $250 \mathrm{~T}$. Let us first identify the differences brought by the addition of a magnetic guide field to the electronic distribution at the vicinity of the bubble boundaries (Figure 1). At the frontier of the bubble, electrons are submitted to two forces. The pondermotive force due to the main pulse repels electrons and thus provides them longitudinal and transverse momenta. Meanwhile, electrons are submitted to the recall electric field induced by the bubble. The balance between these two forces will define the borders of the bubble. When no longitudinal magnetic field is applied electrons flee along straight line trajectories Figure 1(a) around $x=5400$ $c / \omega_{0}$. When a longitudinal magnetic field is added electrons start to revolve around the bubble as a result of the magnetic force. The gyro-radius of the electrons with $p_{\perp} \neq$ 0 (where $p_{\perp}$ denotes the transverse momentum) is reduced when $B_{0}$ is raised. Therefore the corresponding flight path in the $(x, y)$ plane is bent, the trajectory will be even more-curved when the applied field is stronger (Figures 1(b) and (c)).

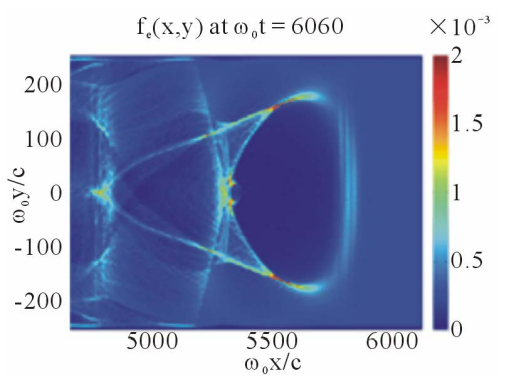

(a)

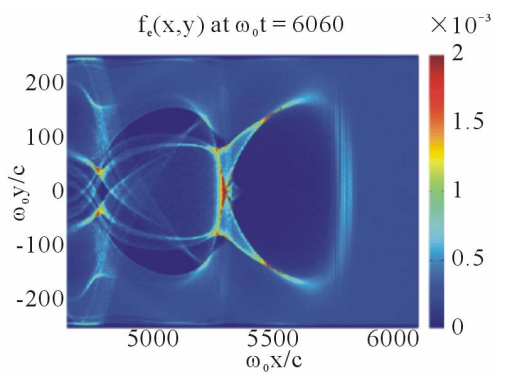

(b)

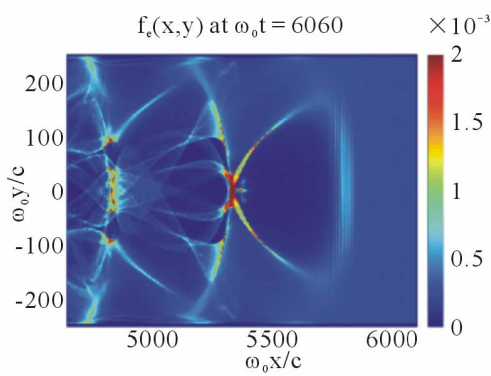

(c)

Figure 1. Electron density long after the collision of the two waves. $\mathrm{P}$ linear polarizations. $a_{0}=4, a_{1}=0.1$ and $n_{e}=2.5 \times 10^{-4} n_{c}$. (a) $B_{0}=0$; (b) $B_{0}=125 \mathrm{~T}$; (c) $B_{0}=250 \mathrm{~T}$. 


\subsection{Self-Consistent Amplification of the Magnetic Field}

When electrons revolve around the bubble they create a current (denoted by $J_{z}$ ) perpendicularly to the plane of the figure, as evidenced by Figure 2. We get a map of the transverse velocity $v_{z}$ when dividing $J_{z}$ by the electronic density $n_{e}$, electrons revolving around the bottle neck of the bubble have velocities in the range $0.5 \leq v_{z} / c$ $\leq 0.8$. This current will act as a small solenoid, and thus the longitudinal magnetic field will be amplified.

The intensity of the magnetic field is almost doubledlocally (Figure 3) compared to the initial $(t=0)$ uniform map of $B_{x}$. We shall underline that this pattern is stable as we obtain quasi identical maps of $B_{x}$ in this re-

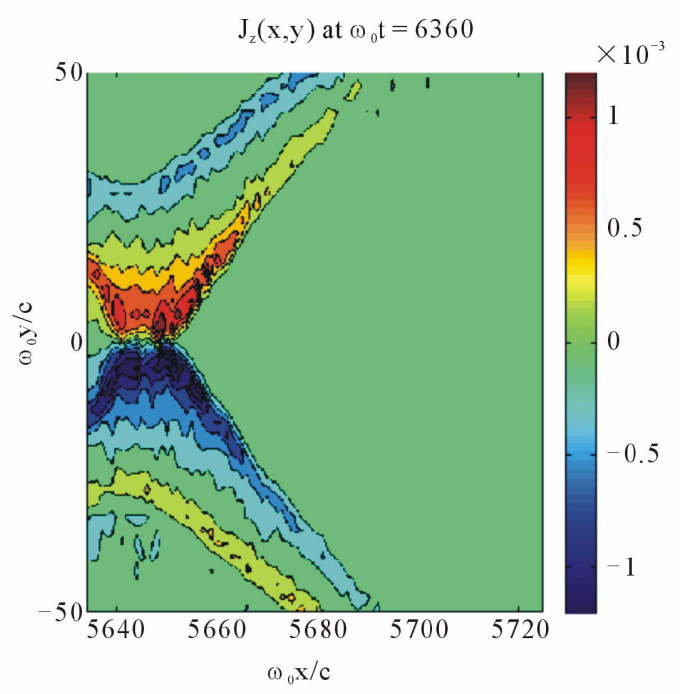

(a) gion of the bubble when the pump pulse has just entered the plasma around $\omega_{0} t=2280$. Moreover we note that the geometry of the magnetic field lines is weakly altered by the electronic density modulations induced by the propagating bubble. Magnetic field lines stay almost parallel to the propagation direction.

\subsection{A Way to Enhance the Beam Emittance}

Let us now examine the effect of the magnetic field on the dynamics of the accelerated beam. The emittance of the trapped beam (i.e. particles with $\gamma>\gamma_{\text {las }}$ ), defined by $\varepsilon_{n, r m s}=\left(1 / m_{e} c\right) \sqrt{\left\langle y^{2}\right\rangle\left\langle p_{y}^{2}\right\rangle-\left\langle y p_{y}\right\rangle^{2}}$, was computed according to Equations (11) and (12) of [17]. The emittance

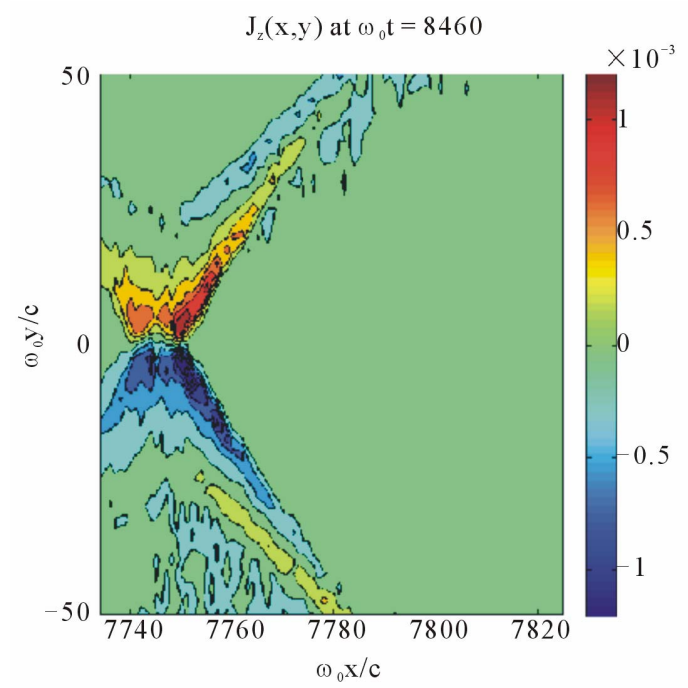

(b)

Figure 2. Transverse component of the current density (normalized by $e_{n} c$ ), the wakefield propagates in a magnetized plasma $\left(B_{0}=250 \mathrm{~T}\right) . a_{0}=4, a_{1}=0.1$ and $n_{e}=2.5 \times 10^{-4} n_{c}$.
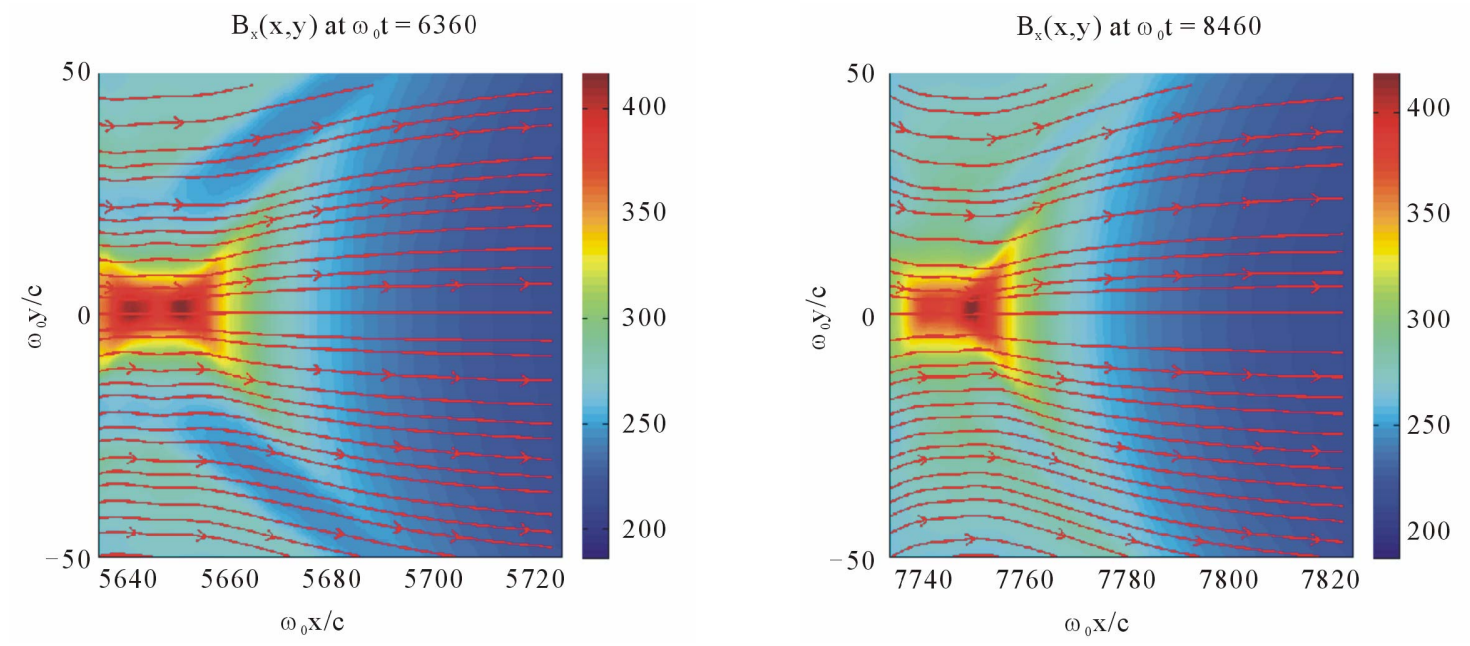

Figure 3. Longitudinal component of the magnetic field (in Teslas), the wakefield propagates in a magnetized plasma $B_{0}=250$ T. $a_{0}=4, a_{1}=0.1$ and $n_{e}=2.5 \times 10^{-4} n_{c}$. Magnetic field lines are superimposed (red curves) on $B_{x}$ color map. 
plot (Figure 4) clearly distinguishes $B_{0}=0, B_{0}=125 \mathrm{~T}$ cases from the highly magnetized case $B_{0}=250 \mathrm{~T}$. In the first category, the emittance slowly grows during the propagation and has a pseudo-periodic oscillation. In the second category, the beam emittance is almost constant during the whole acceleration, and the final value of the beam emittance $\varepsilon_{n, r m s} \sim 0.56 \pi \mathrm{mm}$ mrad is 14 times weaker than in the unmagnetized case! To get more information we have plotted kinetic energy density maps showing the evolution of the trapped beam at the rear of the bubble for both the magnetized and the unmagnetized regimes. In the unmagnetized case (Figure 5), we clearly observe betatron oscillations due to the finite initial radial momentum of injected electrons. Betatron frequency is given by $\omega_{b}=\omega_{p} /(2 \gamma)^{1 / 2}$, according to this formula the pulsation decreases when the beam accelerates. The normalized period $\tilde{T}_{b}=\omega_{0} T_{b}$ minimum value is given by

$$
\tilde{T}_{b}=\frac{2 \pi}{\left(\omega_{b, \max } / \omega_{0}\right)}=\frac{2 \pi}{\left(\left(\frac{\omega_{p}}{\omega_{0}}\right) /\left(2 \gamma_{\text {las }}\right)^{1 / 2}\right)},
$$
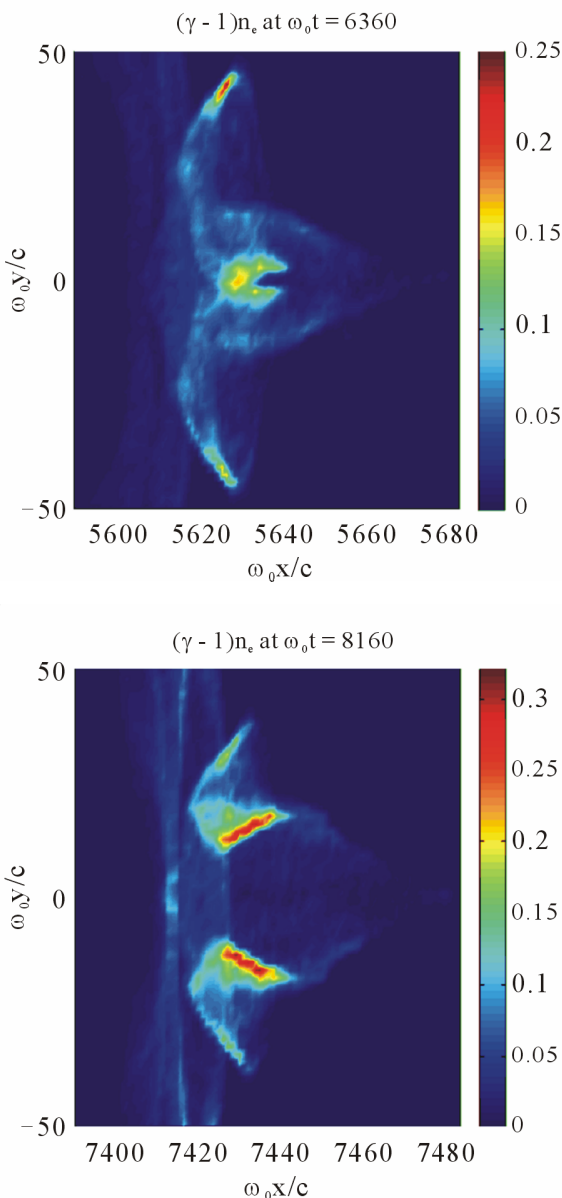

with $\gamma_{\text {las }} \approx 63$ and $n_{e, \text { beam }} \sim 10^{-3} n_{c}$ we get $T_{b} \sim 2200 \omega_{0}^{-1}$. This result is in good agreement with Figure 4 when $B_{0}=$ 0 . The dynamics is completely different in the magnetized

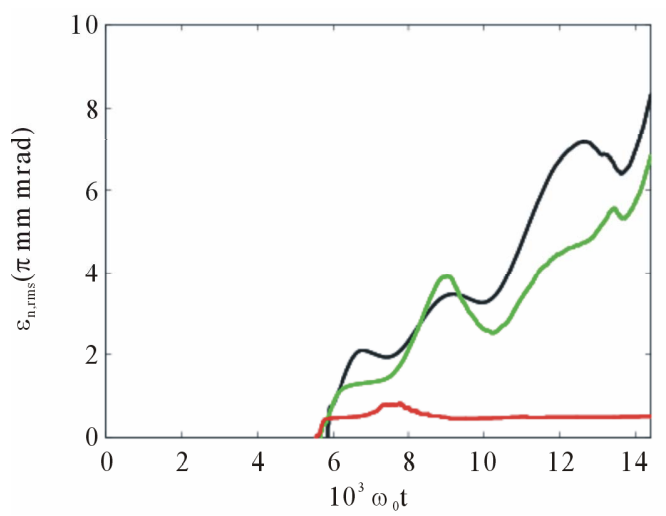

Figure 4. Normalized transverse emittance $\varepsilon_{n, r m s}=\frac{1}{m_{e} c} \sqrt{\left\langle y^{2}\right\rangle\left\langle p_{y}^{2}\right\rangle-\left\langle y p_{y}\right\rangle^{2}}$, in units $\pi$ mm mrad. Black, green and red curves respectively correspond to $B_{0}=0, B_{0}=$ $125 \mathrm{~T}$ and $B_{0}=250$ T. $a_{0}=4, a_{1}=0.1$ and $n_{e}=2.5 \times 10^{-4} n_{c}$.
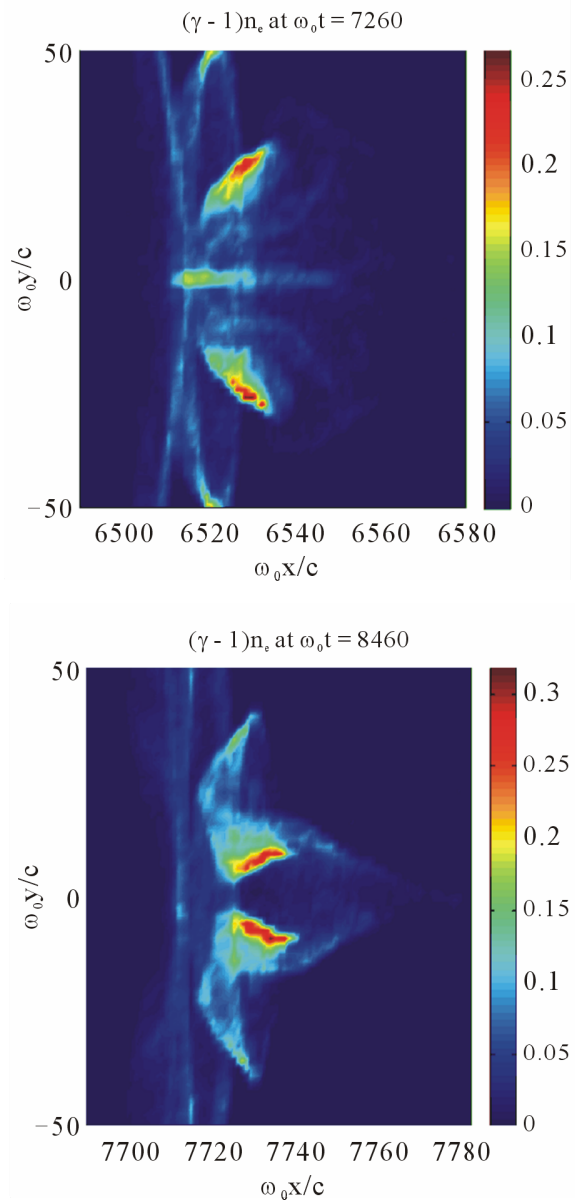

Figure 5. Electron kinetic energy density (normalized by $\left.m_{e} c^{2} n_{c}\right)$, the wakefield propagates in a non magnetized plasma $\left(B_{0}=\right.$ 0). $a_{0}=4, a_{1}=0.1$ and $n_{e}=2.5 \times 10^{-4} n_{c}$. 
case (Figure 6), the longitudinal magnetic field is strong enough to hinder betatron oscillations. As a result the beam is almost concentrated on axis, and the transverse emittance is drastically reduced.

Let us add few words about the magnetization of the plasma. The plasma frequency in the presence of a magnetic field can be approximated by

$$
\omega_{m}=\left(\omega_{e}^{2}+\Omega^{2}\right)^{1 / 2}
$$

where $\omega_{m}$ and $\omega_{e}$ represent the frequencies of the magnetized and unmagnetized plasma, respectively. The cyclotron frequency is defined by $\Omega=e B_{0} / m$. When $B_{0}=$ $125 \mathrm{~T}$ and $B_{0}=250 \mathrm{~T}$, one has

$$
\frac{\Omega^{2}}{\omega_{e}^{2}}=0.34 \text { and } \frac{\Omega^{2}}{\omega_{e}^{2}}=1.38
$$

respectively. We have checked that the modified plasma frequency does not account for the reduction of the beam emittance. For this purpose we have run a simulation, without external field $\left(B_{0}=0\right)$, using a density

$$
\frac{n_{\text {new }}}{n_{c}}=\frac{\left(\omega_{e}^{2}+\Omega^{2}\right)}{\omega_{0}^{2}} \approx 5.95 \times 10^{-4}
$$
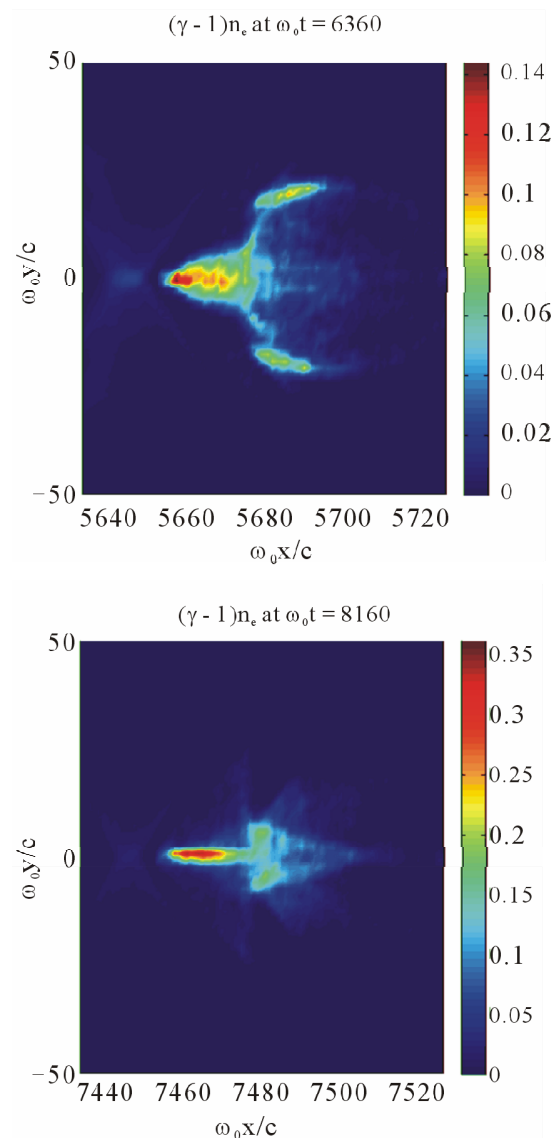

where

$$
\frac{\omega_{e}^{2}}{\omega_{0}^{2}}=\frac{n_{e}}{n_{c}}=2.5 \times 10^{-4} \text { and } \frac{\Omega^{2}}{\omega_{e}^{2}}=1.38 .
$$

The corresponding emittance (Figure 7), even if it is weaker than in our reference case (Figure 4 with $B_{0}=0$ ), exhibits the same slowly growing behavior. This proves that an external magnetic field is necessary to maintain the emittance at low values. In the next section, we will provide some numbers concerning the energy distribution function of the beam.

\section{Influence on the Energy Distribution}

With no guide field, the relative variation of the energy at full width at half maximum (fwhm) $\Delta E_{\mathrm{fwhm}} / E_{\mathrm{max}} \approx 1 \%$ is excellent (Table 1), but the rms (root mean squared) value of the energy spread has small variations and reaches $7 \%$ at the end of the simulation. When $B_{0}=125$ $\mathrm{T}$, on the one hand the spread of the low energy tail of the distribution is reduced as shown by Figure 8(a), and confirmed by the rms value $\sim 4 \%$, but on the other hand $\Delta E_{\mathrm{fwhm}} / E_{\mathrm{max}}$ is slightly degraded. When $B_{0}=250 \mathrm{~T}$, untrapped electrons carrying energies of about $10 \mathrm{Mev}$
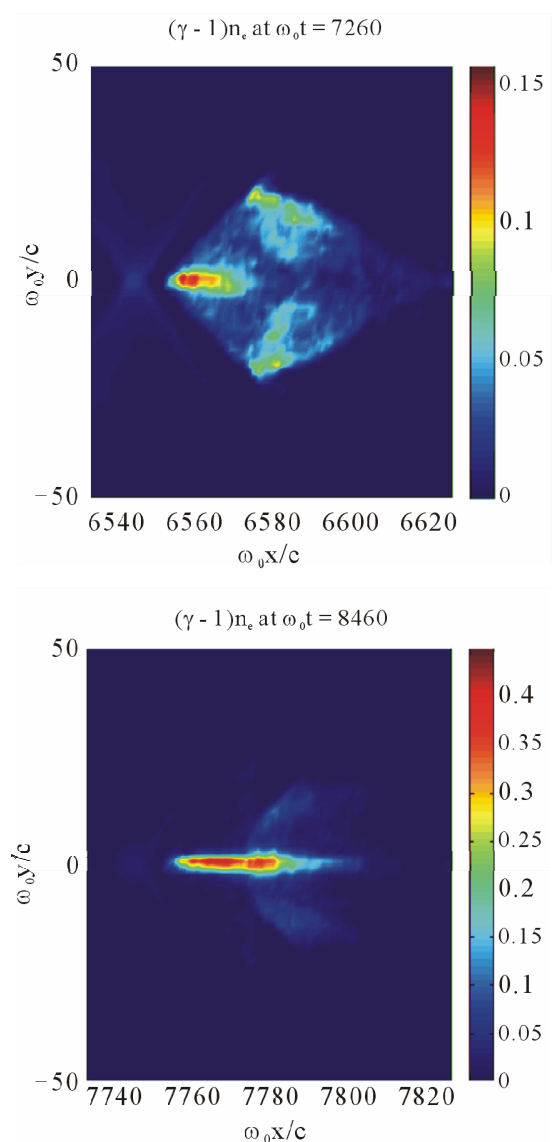

Figure 6. Electron kinetic energy density (normalized by $\left.m_{e} c^{2} n_{c}\right)$, the wakefield propagates in a magnetized plasma $\left(B_{0}=250\right.$ T). $a_{0}=4, a_{1}=0.1$ and $n_{e}=2.5 \times 10^{-4} n_{c}$. 


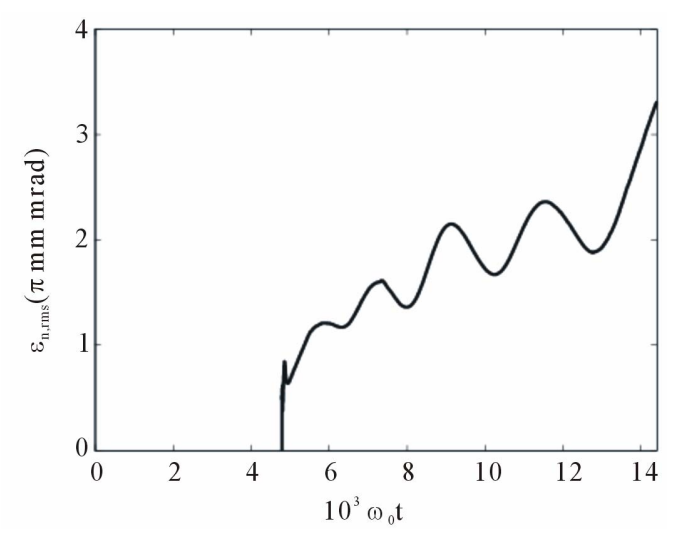

Figure 7. Normalized transverse emittance

$\varepsilon_{n, r m s}=\frac{1}{m_{e} c} \sqrt{\left\langle y^{2}\right\rangle\left\langle p_{y}^{2}\right\rangle-\left\langle y p_{y}\right\rangle^{2}}$, in units $\pi$ mm mrad. $a_{0}=4$, $a_{1}=0.1, n_{\text {new }}=5.95 \times 10^{-4} n_{c}$ and $B_{0}=0$.

Table 1. Evolution of the electron distribution function with $a_{0}=4, a_{1}=0.1, n_{e}=2.5 \times 10^{-4} n_{c}$. Relative variation $\Delta E_{\mathrm{FWHM}} / E_{\max }$ of the distribution, where the subscript FWHM denotes Full Width at Half Maximum.

\begin{tabular}{ccccc}
\hline \multirow{2}{*}{$B_{0}(\mathrm{~T})$} & \multicolumn{4}{c}{$\omega_{0} t$} \\
\cline { 2 - 5 } & 7000 & 9000 & 11000 & 13000 \\
\hline 0 & $2.05 \%$ & $1.09 \%$ & $0.81 \%$ & $0.97 \%$ \\
125 & $5.03 \%$ & $4.89 \%$ & $1.94 \%$ & $1.58 \%$ \\
250 & $3.72 \%$ & $2.75 \%$ & $1.54 \%$ & $0.63 \%$ \\
\hline
\end{tabular}

concentrate $\left(n_{e}\right.$ locally reaches $\left.1.5 \times 10^{-3} n_{c}\right)$ at the rear of the bubble. These low energy (i.e. $0<E_{K}<25 \mathrm{Mev}$ ) electrons are evidenced by bumps in the beam energy distribution (Figure 8(b)). These electrons have energies below the injection threshold and therefore should not be considered for the interpretation of the diagnostics concerning the accelerated beam. The accelerated beam is almost mono-energetic with $\Delta E_{\mathrm{fwhm}} / E_{\max } \leq 1 \%$. Based on this, we may infer that the magnetic field also accounts for the mono-energetic aspect of the energy distribution. To check this pattern we ran other simulations using different waist and duration for the colliding pulse. These two parameters together usually make it possible to get a quasi-mono-energetic electron beam in the blow out regime $[14,18]$. These runs (not shown here) reveal that the energy distribution quality highly depends on beam-loading effects.

The charge injected in the wake strongly depends upon electron trajectories after the lasers have collided, therefore the presence of an external magnetic field will significantly affect the quality of the accelerated beam. Finally the longitudinal magnetic field has no direct effect on the beam energy distribution, but there is an indirect influence as beam-loading is altered. We emphasize that

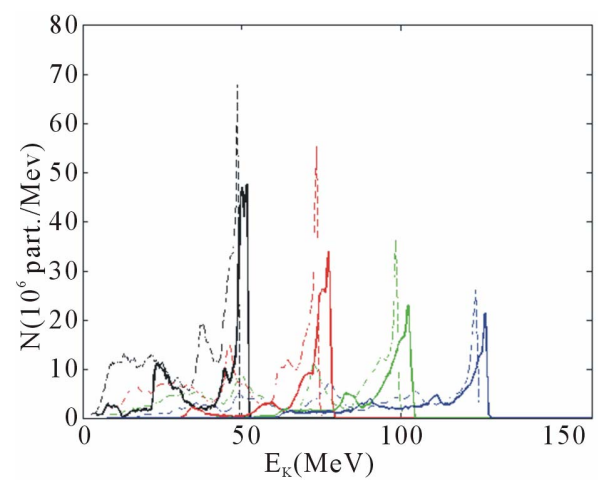

(a)

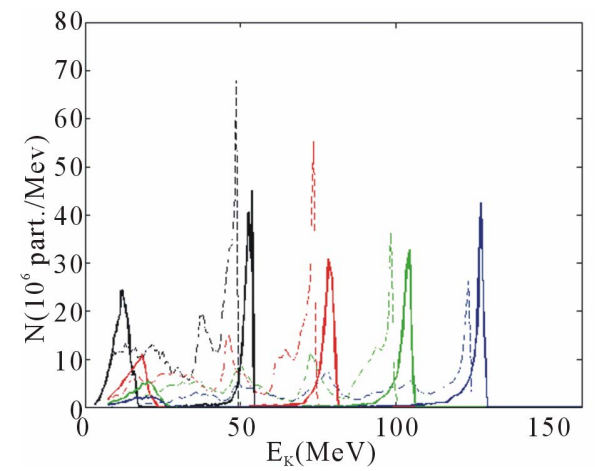

(b)

Figure 8. Electron energy distribution from 2D PIC simulations at $\omega_{0} t=7000,9000,11000,13000$ (black, red, green, blue respectively). $P$ linear polarizations. $a_{0}=4, a_{1}=0.1$ and $n_{e}=2.5 \times 10^{-4} n_{c}$. Dashed lines correspond to $B_{0}=0$. Bold lines correspond to: (a) $B_{0}=125 \mathrm{~T}$; (b) $B_{0}=250 \mathrm{~T}$.

the parametric study we made, by varying the colliding pulse parameters, was conclusive concerning the beam emittance. In fact the emittance of the accelerated beam was always reduced when $B_{0}$ was grown to 125 or 250 $\mathrm{T}$.

\section{Conclusion}

This paper has been devoted to studying the influence of an external magnetic field on the wakefield acceleration process, within the colliding pulse scheme. To our knowledge this idea has never been explored before. The magnetic field is oriented in the direction of propagation of the laser driver. It has been shown that the $B_{0}$ field creates a transverse current, the latter current can induce a raise of $B_{x}$ at the rear bottle neck of the bubble. Therefore the transverse beam dynamics is substantially modified resulting in a considerable reduction of the beam emittance. This mechanism provides means to dramatically enhance the beam quality in the blowout regime. For example with $a_{0}=4, a_{1}=0.1, n_{e}=2.5 \times 10^{-4} n_{c}$ and $B_{0}=250 \mathrm{~T}$ we got $\varepsilon_{n, r m s} \sim 0.56 \pi \mathrm{mm}$ mrad which is more than one order of magnitude better than with no external field $\left(B_{0}=0\right)$. The beam energy distribution is modified 
due to beam-loading effects, in the present paper the parameters of the colliding pulse were adjusted in order to obtain almost the same energy distribution with or without an external magnetic field. Our results should apply in full 3Dx3Dv geometry, however the shape of the bubble may be more significantly altered by the magnetic field than in 2Dx3Dv. In this case it is difficult to tune the parameters in order to get almost the same injected charge with or without external field.

\section{REFERENCES}

[1] T. Tajima and J. M. Dawson, "Laser Electron Accelerator," Physical Review Letters, Vol. 43, No. 4, 1979, pp. 267-270. doi:10.1103/PhysRevLett.43.267

[2] E. Esarey, R. F. Hubbard, W. P. Leemans, A. Ting and P. Sprangle, "Electron Injection into Plasma Wakefields by Colliding Laser Pulses," Physical Review Letters, Vol. 79, No. 14, 1997, pp. 2682-2685. doi:10.1103/PhysRevLett.79.2682

[3] J. Faure, Y. Glinec, A. Pukhov, S. Kiselev, S. Gordienko, E. Lefebvre, J.-P. Rousseau, F. Burgy and V. Malka, "A Laser-Plasma Accelerator Producing Monoenergetic Electron Beams," Nature, Vol. 431, No. 7008, 2004, pp. 541544. doi:10.1038/nature02963

[4] S. P. D. Mangles, C. D. Murphy, Z. Najmudin, A. G. R. Thomas, J. L. Collier, A. E. Dangor, E. J. Divall, P. S. Foster, J. G. Gallacher, C. J. Hooker, D. A. Jaroszinski, A. J. Langley, W. B. Mori, P. A. Norreys, F. S. Tsung, R. Viskup, B. R. Walton and K. Krushelnick, "Monoenergetic Beams of Relativistic Electrons from Intense Laserplasma Interactions," Nature, Vol. 431, No. 7008, 2004, pp. 535-538. doi:10.1038/nature02939

[5] J. B. Rosenzweig, B. Breizman, T. Katsouleas and J. J. Su, "Acceleration and Focusing of Electrons in Two Dimensional Nonlinear Plasma Wakefields," Physical Review A, Vol. 44, No. 10, 1991, pp. R6189-R6192. doi:10.1103/PhysRevA.44.R6189

[6] W. Lu, C. Huang, M. Zhou, W. B. Mori and T. Katsouleas, "Nonlinear Theory for Relativistic Plasma Wakefields in the Blowout Regime," Physical Review Letters, Vol. 96, No. 16, 2006, pp. 165002-1-165002-4. doi:10.1103/PhysRevLett.96.165002

[7] A. Pukhov and J. Meyer-ter-Vehn, "Laser Wakefield Acceleration: The Highly Non-Linear Broken-Wave Regime," Applied Physics B, Vol. 74, No. 4-5, 2002, pp. 355-361. doi:10.1007/s003400200795

[8] S. Gordienko and A. Pukhov, "Scalings for Ultrarelativistic Laser Plasmas and Quasimono Energetic Electrons," Physics of Plasmas, Vol. 12, No. 4, 2005, pp. 043109-1043109-11.
[9] J. Faure, C. Rechatin, A. Norlin, A. Lifschitz, Y. Glinec and V. Malka, "Controlled Injection and Acceleration of Electrons in Plasma Wakefields by Colliding Laser Pulses," Nature, Vol. 444, No. 7120, 2006, pp. 737-739. doi: $10.1038 /$ nature 05393

[10] X. Davoine, E. Lefebvre, J. Faure, C. Rechatin, A. Lifschitz and V. Malka, "Simulation of Quasimonoenergetic Electron Beams Produced by Colliding Pulse Wakefield Acceleration," Physics of Plasmas, Vol. 15, No. 11, 2008, pp. 113102-1-113102-11. doi:10.1063/1.3008051

[11] W. Lu, M. Tzoufras, C. Joshi, F. S. Tsung, W. B. Mori, J. Vieira, R. A. Fonseca and L. O. Silva, "Generating Multi$\mathrm{GeV}$ Electron Bunches Using Single Stage Laser Wakefield Acceleration in a 3D Nonlinear Regime," Physical Review Special Topics-Accelerators and Beams, Vol. 10, No. 6, 2007, pp. 061301-1-061301-12. doi:10.1103/PhysRevSTAB.10.061301

[12] M. S. Hur, D. N. Gupta and H. Suk, "Enhanced ELectron Trapping by a Static Longitudinal Magnetic Field in Laser Wakefield Acceleration," Physics Letters A, Vol. 372, No. 15, 2008, pp. 2684-2687. doi:10.1016/j.physleta.2007.12.045

[13] J. Vieira, S. F. Martins, V. B. Pathak, R. A. Fonseca, W. B. Mori and L. O. Silva, "Magnetic Control of Particle Injection in Plasma Based Accelerators," Physical Review Letters, Vol. 106, No. 22, 2011, pp. 225001-1-225001-4. doi:10.1103/PhysRevLett.106.225001

[14] S. F. Martins, R. A. Fonseca, W. Lu, W. B. Mori and L. O. Silva, "Exploring Laser-Wakefield-Accelerator Regimes for Near-Term Lasers Using Particle-in-Cell Simulation in Lorentz-Boosted Frames," Nature Physics, Vol. 6, No. 4, 2010, pp. 311-316. doi:10.1038/nphys 1538

[15] A. Lagutin, K. Rosseel, F. Herlach, J. Vanacken and Y. Bruynseraede, "Development of Reliable 70 T Pulsed Magnets," Measurement Science and Technology, Vol. 14, No. 12, 2003, p. 2144. doi:10.1088/0957-0233/14/12/015

[16] S. Zherlitsyn, T. Herrmannsdorfer, B. Wustmann and J. Wosnitza, "Design and Performance of Non-Destructive Pulsed Magnets at the Dresden High Magnetic Field Laboratory," IEEE Transactions on Applied Superconductivity, Vol. 20, No. 3, 2010, pp. 672-675. doi:10.1109/TASC.2010.2044158

[17] K. Floettmann, "Some Basic Features of the Beam Emittance," Physical Review Special Topics-Accelerators and Beams, Vol. 6, No. 3, 2003, pp. 034202-1-034202-7. doi:10.1103/PhysRevSTAB.6.034202

[18] X. Davoine, E. Lefebvre, C. Rechatin, J. Faure and V. Malka, "Cold Optical Injection Producing Monoenergetic, Multi-GeV Electron Bunches," Physical Review Letters, Vol. 102, No. 6, 2009, pp. 065001-1-065001-4. doi:10.1103/PhysRevLett.102.065001 